\title{
Percepção de estudantes sobre motivação e aprendizagem em Teoria da Computação com PBL
}

\author{
Luiz Otávio Ramos Gavaza ${ }^{1}$, Laís do Nascimento Salvador ${ }^{1}$, \\ David Moises Barreto dos Santos ${ }^{2}$
}

\author{
${ }^{1}$ Departamento de Ciência da Computação - Universidade Federal da Bahia (UFBA) \\ Av. Adhemar de Barros, S/N, Ondina - 40.170-110 - Salvador - BA - Brasil \\ ${ }^{2}$ Departamento de Ciências Exatas - Universidade Estadual de Feira de Santana (UEFS) \\ Av. Transnordestina, Novo Horizonte - 44.036-900 - Feira de Santana - BA - Brasil \\ \{Igavaza, laisns\}@ufba.br, davidmbs@uefs.br
}

\begin{abstract}
The content of Computer Theory discipline has a high level of abstraction and complexity, and it is an important part of the formation of students in Computer Science courses. Studies has presented evidence that the traditional pedagogical approach has not been certified enough to keep students motivated and able to follow the contents of the subject of Computer Theory. Some constructivist pedagogical approaches have been experienced by educators and researchers in an attempt to improve computer students motivation and learning. This work uses the students' opinion survey to evaluate their perception about motivation and learning in a Computer Theory discipline using the constructivist approach Problem-Based Learning (PBL) in a computer class at the Federal University of Bahia. The results of the experiment showed that the students had good perceptions about the PBL approach, which confirms the potential for the use of PBL in the teaching and learning of Computer Theory discipline.
\end{abstract}

Resumo. O conteúdo de Teoria da Computação possui alto nível de abstração e complexidade, e é parte importante da formação dos estudantes dos cursos de Computação. Estudos levantam indícios de que a abordagem pedagógica tradicional não tem demonstrado ser suficiente para que os estudantes se mantenham motivados e consigam acompanhar os conteúdos da disciplina de Teoria da Computação. Algumas abordagens pedagógicas construtivistas têm sido experimentadas pelos educadores e pesquisadores na tentativa de melhorar a motivação e a aprendizagem dos estudantes de Computação. Este trabalho utiliza pesquisa de opiniões dos estudantes para avaliação da percepção deles sobre a motivação e aprendizagem em uma disciplina de Teoria da Computação com a utilização da abordagem construtivista Aprendizagem Baseada em Problemas (PBL), em uma turma de Computação na Universidade Federal da Bahia. Os resultados da experiência mostraram que os estudantes participantes tiveram boas percepções sobre a abordagem PBL, o que confirma o potencial para utilização de PBL no ensino e aprendizagem da disciplina de Teoria da Computação.

\section{Introdução}

Ao mesmo tempo em que é crescente o número de estudantes universitários, é grande a evasão. Os altos índices de desistências nos cursos superiores são evidências da necessi- 
dade de compreensão das variáveis motivacionais dos estudantes.

A motivação do estudante é um determinante crítico do nível e da qualidade da aprendizagem e do desempenho no contexto escolar. Desta forma, muitos trabalhos têm relacionado o desempenho acadêmico dos estudantes com a motivação [Zenorini et al. 2011, Rufini et al. 2011].

A formação dos estudantes de Computação exige que estes sejam capazes de construir um conhecimento em disciplinas que possuem um alto nível de abstração. A disciplina de Teoria da Computação é uma das disciplinas teóricas com alto nível de abstração nos cursos da área de Computação que exige bastante esforço por parte dos estudantes para que possam acompanhar o andamento, para assim, construírem conhecimento que permita compreender as bases de sustentação da área.

O que percebemos é que a maioria dos cursos da área de Computação utilizam, na maioria das vezes, abordagem de ensino e aprendizagem tradicional, com raras exceções onde são realizadas atividades práticas em laboratórios. Ainda assim, devemos destacar que apenas para algumas disciplinas existem atividades além das aulas expositivas.

A abordagem de ensino e aprendizagem tradicional não tem por objetivo a formação crítica de estudantes, uma habilidade desejável para os estudantes da área de Computação. Além disto, é necessário considerar que na revisão do currículo de Ciências da Computação da Association for Computing Machinery (ACM) em 2013, estão descritas características esperadas para os estudantes de graduação, como a compreensão técnica da ciência da computação, familiaridade com temas e princípios comuns, interação entre teoria e prática, visão sistêmica, habilidades para resoluções de problemas e experiências significativas em projetos, compromisso com a continuidade da aprendizagem, habilidades de comunicação e organização e consciência da aplicabilidade ampla da computação [Sahami et al. 2013].

Existem inúmeras abordagens pedagógicas que poderiam ser utilizas para desenvolvimento crítico dos estudantes e desenvolvimento da habilidades mencionadas na revisão do currículo de Ciências da Computação da ACM em 2013, mas entre as mais promissoras que serviriam as nossas necessidades, sobretudo na possibilidade de desenvolver habilidades de pensamento crítico, se pode destacar a abordagem baseada em problemas.

A abordagem de Aprendizagem Baseada em Problemas, em inglês Problem Based Learning (PBL), foi criada na Universidade McMaster nos anos de 1960 em cursos de medicina, onde é amplamente utilizada [Albanese 2010, Amos and White 1998].

A abordagem PBL pode ser definida como uma abordagem educacional construtivista ativa que usa problemas como contexto para que os estudantes adquiram conhecimentos sobre os conceitos. O foco de aprendizagem está nos estudantes, que são capacitados para que assumam a responsabilidade pela aprendizagem. Nessa abordagem os professores deixam o papel de repositório de conhecimento da abordagem de ensino e aprendizagem tradicional e assumem o papel de tutores atuando como facilitadores da construção do conhecimento [Dolmans et al. 2005, Albanese 2010, Amos and White 1998, Forsythe 2002].

A metodologia baseada em problemas deve capacitar os estudantes a realizar pesquisas, integrar teoria e prática e aplicar conhecimentos e habilidades para desenvolver 
uma solução viável para um problema definido [Savery 2015].

No caso da abordagem baseada em problemas, entre as várias qualidades, podemos destacar que o processo possui como grande objetivo "ensinar a aprender" para que os estudantes possam assumir a responsabilidade pela aprendizagem. Na abordagem baseada em problemas os estudantes são capacitados para as investigações e a aprendizagem dos conceitos será consequência das investigações.

A motivação e qualidade da aprendizagem do estudante é fundamental para que possam construir um conhecimento mais sólido e amplo, investigar outras linhas de estudo e ter opiniões críticas sobre os conceitos. Este trabalho apresenta resultados para uma investigação sobre a percepção de motivação e de aprendizagem de estudantes da disciplina de Teoria da Computação na Universidade Federal da Bahia (UFBA).

Além desta seção introdutória, este trabalho está organizado da seguinte forma: a Seção 2 apresenta o contexto educacional; a Seção 3 para descrever a metodologia; a Seção 4 para apresentar os trabalhos correlatos; a Seção 5 para trazer uma discussão sobre alguns dos resultados obtidos com a experiência; por fim, a Seção 6 para trazer algumas das argumentações sobre consequências dos resultados exibidos na Seção anterior, as expectativas e as possibilidades de trabalhos na investigação proposta.

\section{Contexto Educacional}

Os cursos de Computação na UFBA utilizam majoritariamente a abordagem tradicional, onde algumas disciplinas específicas contam com carga horária específica para laboratórios práticos. Existem algumas poucas iniciativas de novas abordagens como é o caso deste estudo.

A disciplina em que este estudo foi realizado, apesar de introdutória, trabalha com diversos dos conceitos da Teoria da Computação, incluindo a Teoria da Complexidade e dos Compiladores.

Os estudantes estão entre o terceiro e o quinto semestre de um curso noturno em uma universidade pública, são majoritariamente do sexo masculino e possuem idade entre 19 e 44 anos.

A carga horária de 68 horas da disciplina no semestre foi dividida em: 26 horas para a realização de sessões tutoriais de PBL em que foi conduzida a abordagem; 32 horas para a realização de aulas expositivas em que o educador apresentou conceitos da disciplina; e 10 horas para a realização de avaliações tradicionais.

O conteúdo da disciplina, além dos problemas, foi trabalhado com aulas expositivas e construção de um projeto de desenvolvimento. A avaliação de conceito dos estudantes foi realizada com atribuição de notas para o projeto de desenvolvimento para os problemas e duas avaliações escritas individuais e sem consultas. No caso dos problemas, a nota considerou assiduidade e participação dos estudantes nas discussões das sessões tutoriais e o produto produzido como solução para o problema.

A condução da disciplina estimulou os estudantes a utilizarem um ambiente virtual de aprendizagem (AVA) institucional para continuidade das discussões sobre os problemas e conceitos em fóruns, além de repositório para armazenar os conteúdos produzidos. 


\section{Metodologia}

No estudo foi utilizada uma metodologia de pesquisa descritiva na forma de pesquisa de opinião que contou com a participação de 50 estudantes que foram selecionados por oportunidade de estarem inscritos na disciplina em que este estudo foi realizado.

A experiência aconteceu em uma sala de aula tradicional equipada com um quadro branco e com quadros adicionais feitos com papel metro colados nas paredes da sala. Nas sessões tutorias os estudantes foram distribuídos em grupos de cinco até dez participantes. Os grupos formaram semicírculos de forma que todos foram capazes de enxergar o quadro adicional referente ao grupo ao qual foram designados e tiveram interação dentro do grupo facilitada.

A metodologia de pesquisa realizou aplicações de cinco problemas PBL no semestre 2017.1 que foram construídos baseados em sete princípios apresentados por Dolmans et al. (1997).

A abordagem PBL foi exemplificada para os estudantes utilizando um problema que foi construído especificamente com este propósito, o problema "A torta e o ladrão de pimenta". Podemos destacar que este problema trabalha os conceitos básicos de inferência lógica, assim, poderia também utilizado nesse contexto de ensino e aprendizagem. Uma cópia está disponível em https://goo.gl/5DD83a.

Os problemas inicialmente fornecem aos estudantes pequenos desafios e a cada novo problema tem um aumento gradual na complexidade em relação ao anterior, que é obtido removendo orientações específicas, assim, os problemas apresentam cada vez mais características dos problemas encontrados no mundo real [Fee and Holland-Minkley 2010].

Segue a lista dos problemas:

- Problema 1 - O código Morse - O problema menciona um grupo de estudantes programadores que estão naufragados em uma ilha deserta e precisam submeter um projeto de desenvolvimento. Ao serem resgatados devem discutir como utilizar um telégrafo para realizar a transmissão.

O objetivo desse problema é trabalhar os conceitos de Linguagens Formais por meio de uma equivalência com o código Morse.

Disponível em https://goo.gl/M9r9oA

- Problema 2 - A máquina de vender refrigerantes - O problema menciona uma empresa que deseja construir uma máquina de vender refrigerantes.

O objetivo desse problema é trabalhar os conceitos de Autômatos Finitos e introduzir o conceito de não determinismo.

Disponível em https://goo.gl/gGg1Ch

- Problema 3 - O controle de tráfego - O problema menciona a situação de buracos em uma estrada e solicita aos estudantes que realizem o balanceamento da proporção entre veículos leves e pesados.

O objetivo desse problema é trabalhar os conceitos de Autômatos Finitos com Pilha.

Disponível em https://goo.gl/BcTJ7w

- Problema 4 - O controle de tráfego - O problema é uma extensão do Problema 3.

O objetivo é trabalhar os conceitos de Máquinas de Turing.

Disponível em ttps://goo.gl/2LXKku 
- Problema 5 - Uma função bastante curiosa - O problema relata sobre a capacidade dos compiladores em identificar erros e otimizar códigos e convida os estudantes a discutirem o quanto um compilador pode ser inteligente.

O objetivo é trabalhar o Problema da Parada.

Disponível em https://goo.gl/rUyNov

O conteúdo da Teoria da Complexidade, que está presente entre os conteúdos da disciplina em que aplicamos a metodologia, foi trabalhado com um problema em que os estudantes discutiram nos mesmos moldes da abordagem PBL, entretanto, ao invés de apresentarem um produto como solução, eles responderam uma lista de questões. Este problema foi utilizado no critério de avaliação dos estudantes. Uma cópia está disponível em https://goo.gl/bewLVy.

Para trabalhar os conceitos de Teoria dos Compiladores foi utilizado um projeto de implementação de uma calculadora pós-fixa. Uma cópia da especificação está disponível em https://goo.gl/6zW9Wh.

Para cada problema os estudantes foram convidados a responder no AVA um formulário de percepções com itens de caracterização de perfil, com afirmativas em escala Likert de cinco níveis e com um espaço aberto. Para evitar que dados da pesquisa modificassem a condução da metodologia, os dados foram processados apenas ao final de toda coleta. A assinatura do termo de consentimento livre e esclarecido pelos participantes foi digital, no AVA.

\section{Trabalhos correlatos}

Os educadores e pesquisadores têm buscado alternativas pedagógicas para motivação e aprendizagem dos estudantes em disciplinas de Computação e têm relatado as experiências, assim como é realizado neste trabalho.

A utilização mecânica de conceitos de Teoria da Computação por parte dos estudantes é a justificativa para a introdução de abordagens construtivistas no trabalho Chesnevar et al. (2004). Neste trabalho uma série de abordagens construtivistas foram combinadas e aplicadas por três semestres. A principal conclusão do trabalho é que os temas da disciplina de Teoria da Computação podem se tornar mais interessante se for utilizada uma combinação de diferentes abordagens. Entendemos que assim como em nosso trabalho, apesar de neste não está explicito, também foi realizada uma pesquisa de percepção com os estudantes, que também apresentou elevado índice de satisfação com abordagens construtivistas, mas a quantificação da satisfação não foi apresentada em resultados.

A utilização de uma ferramenta de aprendizagem é a proposta pedagógica em Vieira et al. (2003), onde é construída uma ferramenta denominada de Language Emulator onde os estudantes podem utilizar expressões regulares, gramáticas regulares e autômatos finitos. O principal destaque deste trabalho é que os estudantes podem obter respostas tempestivamente, assim, isto pode contribuir no processo de motivação, entretanto, apesar disto, e de ter obtido uma aprovação de $95 \%$ dos participantes, apenas alguns dos conceitos de Teoria da Computação estão contemplados pela ferramenta.

Os jogos educacionais são propostas comuns para abordagem pedagógica, e algumas são as propostas que utilizam desta alternativa para motivação e aprendizagem de 
estudantes em disciplinas de Computação. Os aspectos lúdico e interativo dos jogos educacionais pode ser uma boa alternativa para auxiliar na aprendizagem e motivação dos estudantes [Silva et al. 2010].

O trabalho Leite et al. (2014) propõe a utilização de um jogo educacional com um ambiente para correção automática de exercícios de Teoria da Computação. Apesar de bem avaliada pelos estudantes, a ferramenta contempla apenas uma parte dos temas da disciplina de Teoria da Computação.

A utilização de um jogo educacional também é a proposta descrita em de Morais et al. (2011) como abordagem pedagógica para motivação dos estudantes e aprendizagem dos conteúdos da disciplina de Teoria da Computação. A ideia de os estudantes terem um aplicativo móvel permite que eles tenham acesso facilitado aos conceitos da disciplina de Teoria da Computação a qualquer momento, entretanto, neste trabalho o jogo apresentado parece uma lista de exercícios e a única história, sem diversos caminhos possíveis, pode não ser suficiente para motivação dos estudantes.

O curso de Engenharia da Computação na Universidade Estadual de Feira de Santana utiliza uma abordagem com PBL desde a criação do curso em 2003. A escolha pela utilização da abordagem baseadas em problemas ocorreu desde a elaboração do Projeto de curso. A flexibilidade é uma das principais características do curso, onde é permitido aos estudantes não só dentro das disciplinas escolherem por caminhos diversos para a solução dos problemas e construção do conhecimento, mas também na forma como este estudante vai integralizar o seu currículo para formação. Para aplicação da abordagem PBL, no curso da UEFS, são utilizadas infraestruturas específicas [dos Santos et al. 2007, Bittencourt and Figueiredo 2003]. O curso possui dez módulos curriculares em que são aplicados a metodologia PBL, entre eles podem ser citados Estruturas de Dados e Engenharia de Software. Apesar de consolidado o método PBL na UEFS, não há aplicação da metodologia PBL para a disciplina de Teoria da Computação [dos Santos and Burnham 2003].

\section{Resultados}

Apesar de ser uma disciplina situada no início do curso, entre os estudantes participantes deste estudo é grande a quantidade de estudantes que já abandonaram uma outra disciplina do curso. A disciplina deste estudo também possui historicamente um índice elevado de evasão. Para exemplificar sobre a evasão, utilizando uma abordagem tradicional com aulas expositivas, a disciplina teve 14 estudantes desistentes em um total de 28 estudantes matriculados no semestre 2014.1, e 10 estudantes desistentes em 24 estudantes matriculados no semestre 2015.1.

Como a participação nas pesquisas foi opcional, a partir deste ponto, o termo "participante" é utilizado para designar apenas o estudante que respondeu a pesquisa mencionada na discussão, não devendo ser confundido com o termo "estudante", que a partir deste ponto é utilizado para designar o participante da metodologia, independente de participação na pesquisa.

Os resultados apresentados neste trabalho utilizaram as respostas dos participantes nas afirmativas de percepção sobre motivação e aprendizagem. A apresentação dos resultados utiliza gráficos dispostos em barras, que foram construídas de forma a facilitar a leitura por nível de concordância para cada aplicação de problema. Cada uma das 
barras representa uma aplicação, que está identificada conforme a numeração utilizada na Seção 3. A concordância pode ser lida por porcentagem de satisfação, em $y_{1}$ à esquerda, ou adicionalmente, por porcentagem de insatisfação em $y_{2}$ à direta.

Para as discussões e conclusões apresentadas neste trabalho será avaliada a concordância na percepção dos participantes. As percepções com algum nível de concordância positiva, isto é, o participante respondeu "concordo" ou "concordo parcialmente" para a afirmativa será considerada com avaliação favorável.

A Figura 1 exibe um gráfico com os resultados de percepção dos participantes para a motivação nas cinco aplicações de problemas deste trabalho. A informação é obtida com a extração de dados das respostas dos participantes para a afirmativa "O problema lhe deixou motivado para descobrir uma possível solução”.

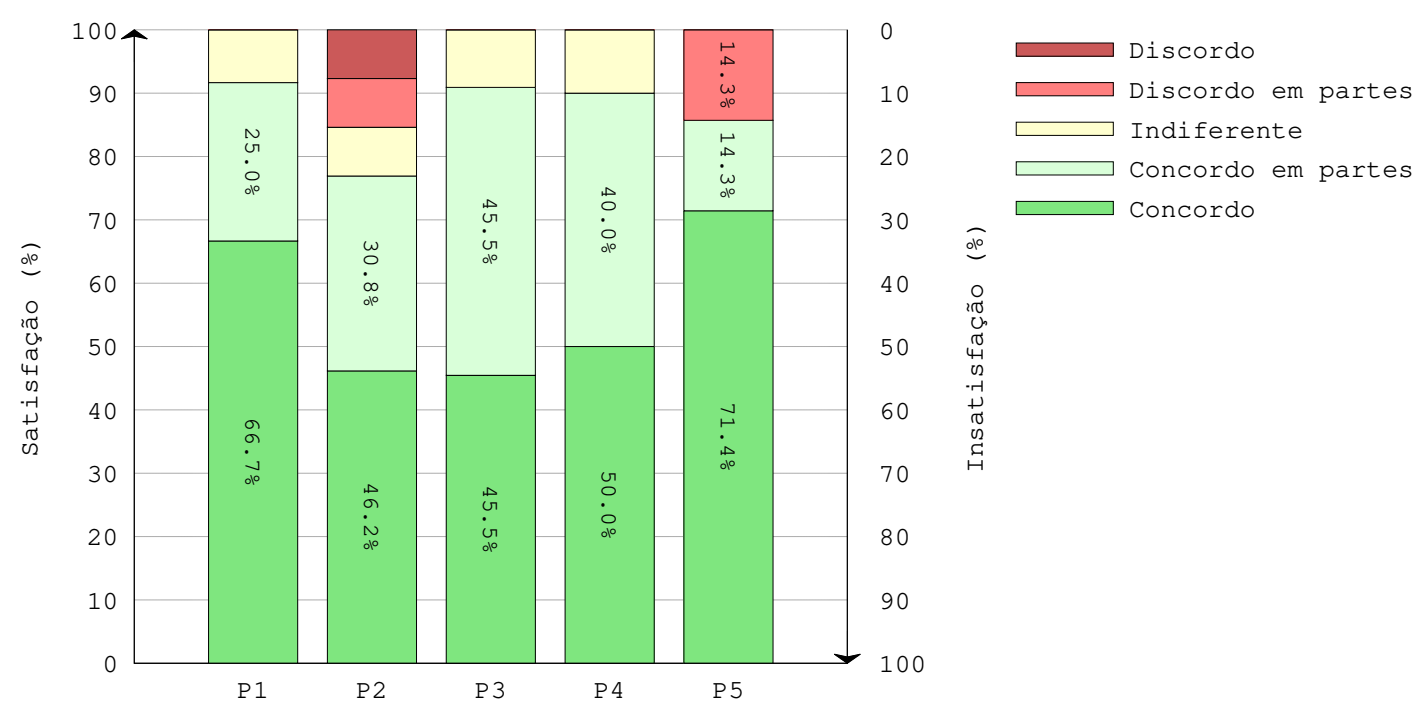

Figura 1. Percepções dos participantes sobre motivação

Em todas as aplicações dos problemas a percepção dos participantes para a motivação obteve bons resultados. A avaliação menos favorável, na aplicação do problema "A máquina de vender refrigerantes", obteve satisfação com relação à percepção de motivação por parte de $77,0 \%$ dos participantes. Em três das cinco aplicações ao menos $90,0 \%$ dos participantes se sentiram motivados.

A Figura 2 exibe um gráfico com os resultados de percepção dos participantes para a aprendizagem nas cinco aplicações deste trabalho. A informação é obtida com a extração de dados das respostas dos participantes para a afirmativa "Você acredita que cumpriu com os objetivos de aprendizagem do problema".

A percepção de aprendizagem também obteve bons resultados em todas as replicações. A avaliação menos favorável, na aplicação do problema "Uma função bastante curiosa", obteve satisfação com relação à percepção de aprendizagem por parte de $57,2 \%$ dos participantes. Em três das cinco replicações ao menos $80,0 \%$ dos participantes acreditaram ter cumprido com os objetivos de aprendizagem.

É possível perceber diferenças entre os resultados com relação à percepção de aprendizagem pelos participantes nas replicações. Uma alternativa é discutir essas 


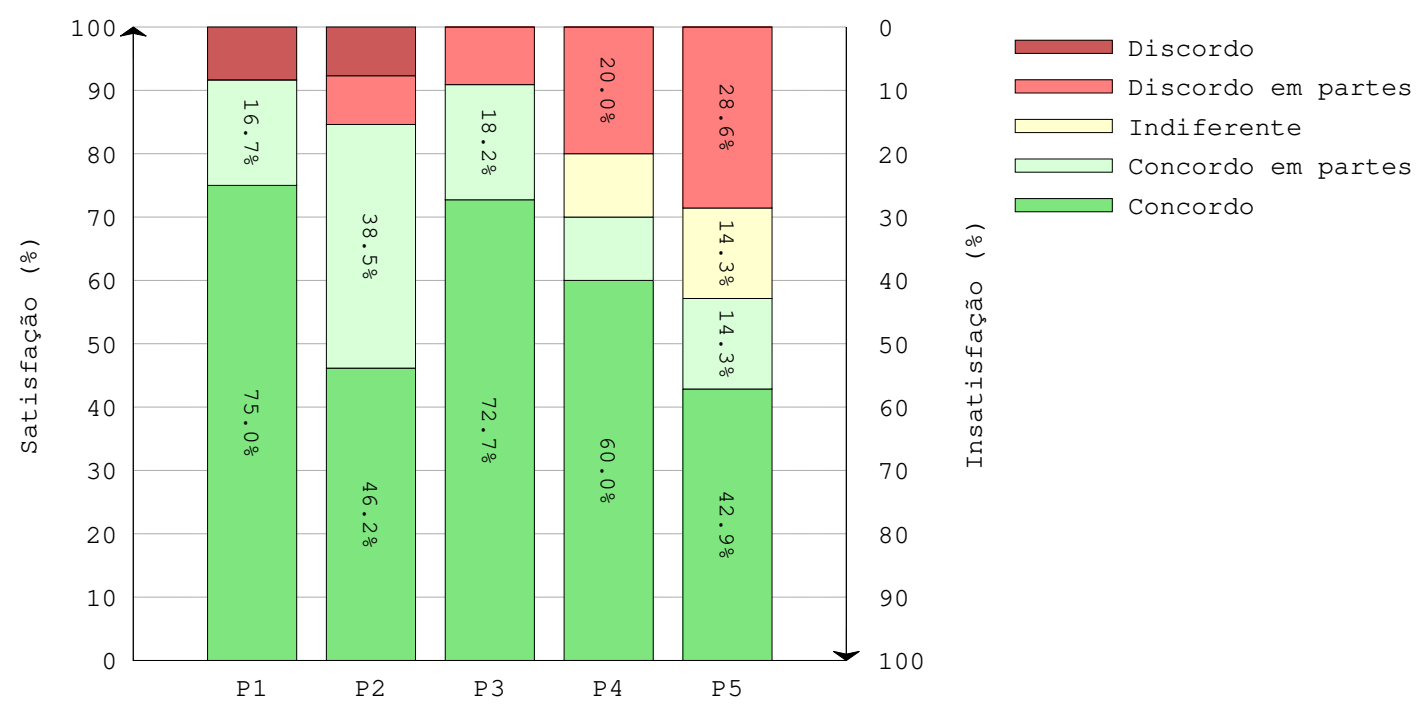

Figura 2. Percepções dos participantes sobre aprendizagem

diferenças do ponto de vista da crescente dificuldade e abstração dos conteúdos dos problemas. O processo de incremento da dificuldade ou desafio nos problemas pode não funcionar perfeitamente em uma sala de aula tradicional com todas as dificuldades que conhecemos, por este motivo, a transição entre os problemas pode não amortizar suficientemente o incremento de dificuldade e nível de abstração necessários para entendimento dos conceitos, assim, os estudantes podem ter sentido uma ruptura, como consequência, a percepção com relação à aprendizagem é apresentada com uma tendência de redução.

Os participantes inclusive mencionaram sobre as dificuldades com os conceitos da disciplina no espaço aberto da aplicação do problema "Uma função bastante curiosa".

Os comentários em relação à metodologia PBL foram na maioria elogiosos. Foi mencionado que a metodologia ajuda no processo de aprendizagem da disciplina, além de ser interessante e motivadora.

A aprendizagem mais profunda dos conteúdos e a necessidade de mais esforço por parte dos estudantes também foram destacadas. Acreditamos que nesse contexto, o objetivo de fazer com que os estudantes assumam também a responsabilidade pela a sua aprendizagem fica explicitada.

\section{Conclusão}

Os bons resultados da experiência deste estudo podem ser utilizados como argumentação da possibilidade de utilização da abordagem baseada em problemas para motivação e aprendizagem de estudantes em disciplinas teóricas de Computação.

Nessa experiência percebemos que alguns estudantes possuem um perfil mais passivo de aprendizagem ou possuem outras obrigações que é utilizada por estes como justificativa para uma menor dedicação em buscar conhecimento, nesse contexto, traçar um perfil mais detalhado dos estudantes pode permitir a construção de problemas que estejam mais alinhados com estes perfis.

A motivação e absorção de conhecimento pelos estudantes deve ser avaliada com- 
parativamente com outras abordagens, inclusive a abordagem pedagógica tradicional. Também é necessário uma avaliação sobre a absorção de conhecimento além da percepção dos estudantes, por exemplo, verificar se a utilização da abordagem baseada em problemas é capaz de melhorar o desempenho dos estudantes em avaliações tradicionais.

No que diz respeito a motivação dos estudantes, se faz necessário realizar futuramente uma análise que realize cruzamento com dados de evasão. A identificação dos principais motivos para evasão dos estudantes pode permitir ações efetivas na reversão da evasão, inclusive contribuir na construção de problemas mais efetivos para utilização de PBL como ferramenta para redução de evasão.

Estudar as correlações entre variáveis como percepção de motivação e percepção de aprendizagem também é um trabalho futuro. Nesse estudo eventualmente pode surgir um importante indicador para o educador acompanhar o envolvimento e desenvolvimento dos estudantes.

Além das contribuições diretas nos resultados, como na discussão do potencial da abordagem PBL para o ensino e aprendizagem de disciplinas teóricas de Computação, este trabalho também trouxe diversas questões de estudos futuros.

\section{Agradecimentos}

Os autores deste trabalho gostariam de agradecer aos revisores anônimos deste trabalho pelas contribuições que ajudaram a melhorar a versão final deste artigo.

\section{Referências}

Albanese, M. A. (2010). Problem-based learning. In An introduction to medical teaching, pages 41-53. Springer.

Amos, E. and White, M. J. (1998). Problem-based learning. Nurse Educator, 23(2):1114.

Bittencourt, R. A. and Figueiredo, O. A. (2003). O currículo do curso de engenharia de computação da uefs: Flexibilização e integração curricular. In XI Workshop sobre Educação em Computação-Anais do XXIII Congresso da Sociedade Brasileira de Computação-Anais do, pages 171-182.

Chesnevar, C. I., González, M. P., and Maguitman, A. G. (2004). Didactic strategies for promoting significant learning in formal languages and automata theory. ACM SIGCSE Bulletin, 36(3):7-11.

de Morais, D. C. S., Alencar, A. D., and de Souza, R. (2011). Jogo baseado em mlearning e aprendizado tangencial para auxílio ao ensino de teoria da computação. In Brazilian Symposium on Computers in Education (Simpósio Brasileiro de Informática na Educação-SBIE), volume 1.

Dolmans, D. H., De Grave, W., Wolfhagen, I. H., and Van Der Vleuten, C. P. (2005). Problem-based learning: Future challenges for educational practice and research. $\mathrm{Me}$ dical education, 39(7):732-741.

Dolmans, D. H., Snellen-Balendong, H., and van der Vleuten, C. P. (1997). Seven principles of effective case design for a problem-based curriculum. Medical Teacher, 19(3):185-189. 
dos Santos, D. M. B. and Burnham, T. F. (2003). O pensamento de paulo freire e pbl: primeiras aproximações e afastamentos. In XI Workshop sobre Educação em Informática (WEI 2003), Campinas, SP.

dos Santos, D. M. B., Pinto, G., Sena, C. P. P., Bertoni, F. C., and Bittencourt, R. A. (2007). Aplicação do método de aprendizagem baseada em problemas no curso de engenharia da computação da universidade estadual de feira de santana. In Congresso Brasileiro de Educação em Engenharia-COBENGE.

Fee, S. B. and Holland-Minkley, A. M. (2010). Teaching computer science through problems, not solutions. Computer Science Education, 20(2):129-144.

Forsythe, F. (2002). Problem-based learning. The Handbook for Economics Lecturers: Teaching, Bristol: Economics LTSN, http://www. economicsnetwork. ac. uk/handbook.

Leite, L. S., Sibaldo, M. A. A., Carvalho, T., and Souza, R. (2014). Montanha de chomsky: jogo tutor para auxílio no ensino de teoria da computação. In XXII Workshop sobre Educação em Informática (WEI 2014), Brasília, DF.

Rufini, S. É., Bzuneck, J. A., and de Oliveira, K. L. (2011). Estudo de validação de uma medida de avaliação da motivação para alunos do ensino fundamental. Psico-USF, 16(1):1-9.

Sahami, M., Roach, S., Cuadros-Vargas, E., and LeBlanc, R. (2013). Acm/ieee-cs computer science curriculum 2013: reviewing the ironman report. In Proceeding of the 44th ACM technical symposium on Computer science education, pages 13-14. ACM.

Savery, J. R. (2015). Overview of problem-based learning: Definitions and distinctions. Essential readings in problem-based learning: Exploring and extending the legacy of Howard S. Barrows, 9:5-15.

Silva, R. C., Binsfeld, R. L., Carelli, I. M., and Watanabe, R. (2010). Automata defense 2.0: reedição de um jogo educacional para apoio em linguagens formais e autômatos. In Brazilian Symposium on Computers in Education (Simpósio Brasileiro de Informática na Educação-SBIE), volume 1.

Vieira, L. F. M., Vieira, M. A. M., and Vieira, N. J. (2003). Language emulator, uma ferramenta de auxílio no ensino de teoria da computação. In XIII Workshop sobre Educação em Computação-XXV Congresso da Sociedade Brasileira de Computação.

Zenorini, R. d. P. C., Santos, A. A. A. d., and Monteiro, R. d. M. (2011). Motivação para aprender: relação com o desempenho de estudantes. Paidéia, 21(49):157-164. 